\title{
8.3 LOW MODE COHERENT SYNCHROTRON RADIATION AND PULSAR MODELS
}

\author{
BERNARD J. EASTLUND
}

Division of Research, U.S. Atomic Energy Commission, Washington, D.C., U.S.A.

\begin{abstract}
The radio pulses from pulsars are considered to be formed from the beamed radiation from relativistic electrons. This radiation is in low order harmonics of the gyro frequency, giving pulse shapes and polarisations which agree with observation.

A possible connection between the shapes of radio pulses and the emission of optical pulses, via the electron energy, leads to a suggested means of searching for visible pulsars with improved sensitivity.
\end{abstract}

The theory for pulsar radiation described in the present paper is based on a model in which an oblique rotator emits coherent synchrotron radiation. In this model, illustrated in Figure 1, the knife-like angular pattern of coherent synchrotron radiation from charged particles trapped in a magnetosphere is converted into a time dependent pulse at the location of an observer by rotation of the star.

Work with this model to date (Maran and Cameron, 1968; Eastlund, 1968, 1970a, b) has concentrated on a comparison of individual and average RF pulses with a detailed computation of pulse shapes based on the well known single particle synchrotron emission formula given by Equation (1) (Bekefi, 1966).

$$
P_{m}=\frac{e^{2} m^{2} \omega_{L}^{2}}{8 \pi \varepsilon_{0} c}\left[\left(\frac{\cos \theta-\beta_{\|}}{\sin \theta}\right)^{2} J_{m}^{2}\left(m \beta_{\perp} \sin \theta\right)+\beta_{\perp}^{2} J_{m}^{\prime 2}\left(m \beta_{\perp} \sin \theta\right)\right]
$$

where $\quad P_{m}=$ the intensity of synchrotron mode, $m$.

$$
\begin{aligned}
\omega_{L} & =\frac{m \omega_{0}}{\left(1-\beta_{\|} \cos \theta\right)} \\
\beta_{\perp} & =v_{\perp} / c \\
\beta_{\|} & =v_{\|} / c \\
m_{0} & =\text { mass of the charged particle } \\
\gamma & =\left(1-\beta^{2}\right)^{-1 / 2} \\
\theta & =\text { the angle between the observer and the magnetic field direction. }
\end{aligned}
$$

The transformation between angular dependence and time dependence is made using the following expression:

$$
P_{m}(\theta)=P_{m}\left(\frac{2 \pi}{\tau} t\right)
$$

where $\tau$ is the pulse repetition rate.

For relativistic electrons, Nodvick and Saxon (1954) have shown that the single 


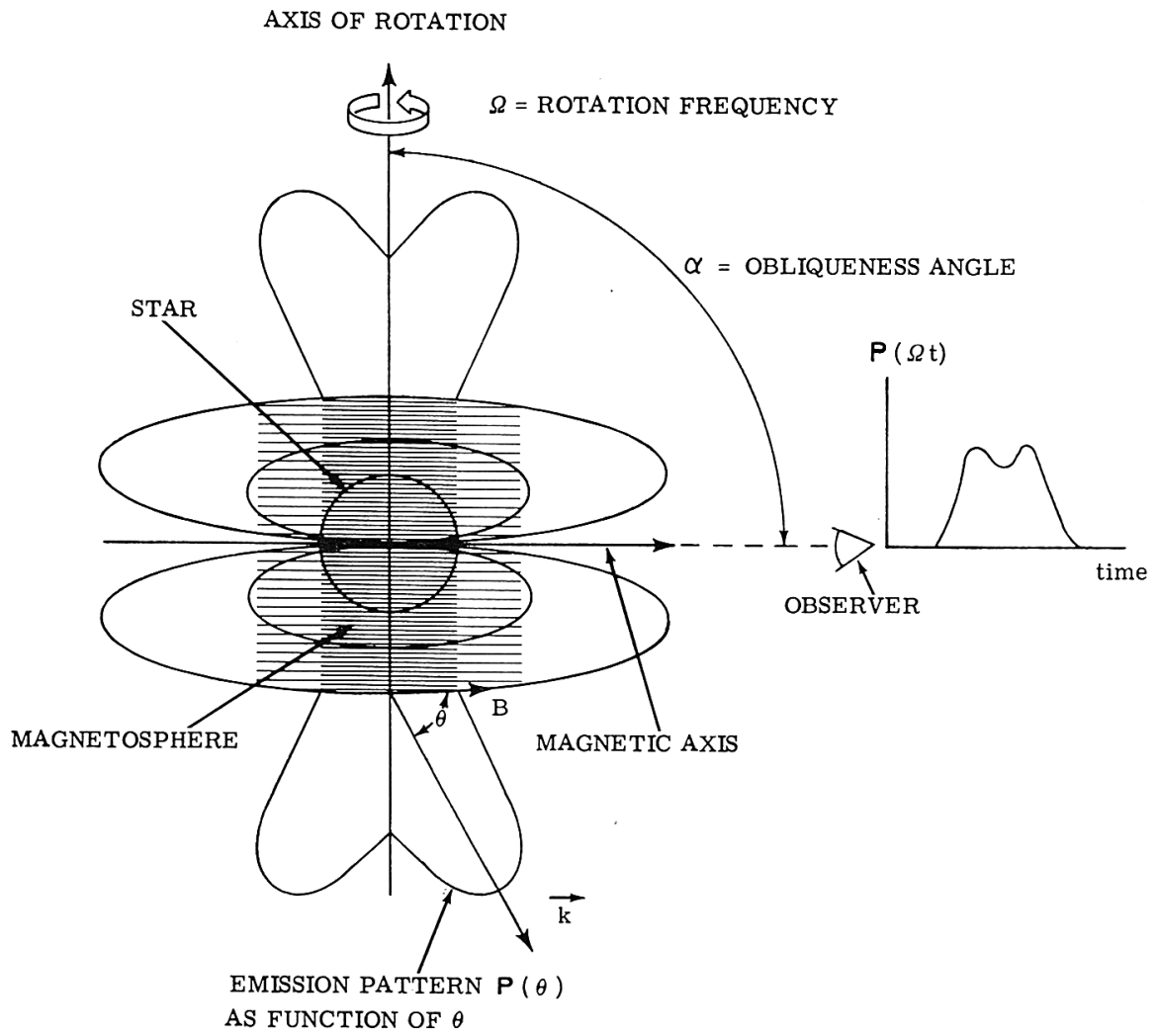

Fig. 1.

particle spectrum of Equation (1) is related to the intensity of coherent radiation as follows:

$$
P=N^{2} \sum_{m=1}^{\infty} P_{m} f_{m}
$$

where $\quad f_{m}=e^{-m^{2} \psi^{2} / 4}$

$N=$ the number of electrons phase bunched over an angle $\psi$ on a cyclotron orbit.

This equation is presented to allow some discussion of the expected coherent synchrotron spectrum and its relation to optical spectra. It is assumed that details of the magnetosphere and an exact calculation of the coherent effect will be much more complicated than the prediction of Equation (3).

To conserve time and permit a more detailed presentation of new results the previous work based on the above equations will not be described in detail in this paper. 
According to Equation (1), the pulse shape is a function of the synchrotron mode number, $m$; the components of the particle velocity perpendicular, $\beta_{\perp}$ and parallel, $\beta_{\|}$to the magnetic field. The value of the magnetic field does not effect the pulse shape. Figure 2 shows the predicted pulse intensity and polarization (Stokes parameters $I$, $L=\left(Q^{2}+U^{2}\right)^{1 / 2}$ and $V$ are calculated assuming an obliqueness angle $\alpha=90^{\circ}$ with an observer located in the star's equatorial plane) for fixed particle energy ( $8 \mathrm{MeV})$ and synchrotron mode $m=110$ with variable $\beta_{\|}$. (Mode 110 corresponds roughly to a receiver frequency of $25 \mathrm{MHz}$ based on Figure 7 of Eastlund, 1970a.) Note the asymmetry in the pulse shapes as $\beta_{\|}$increases. There is evidence of such asymmetric behavior in data from many pulsars (Clark and Smith, 1969; Drake and Craft, 1968). Detailed study of such behavior may indicate the mirroring of particles trapped in the pulsar magnetosphere (Eastlund, 1970b).
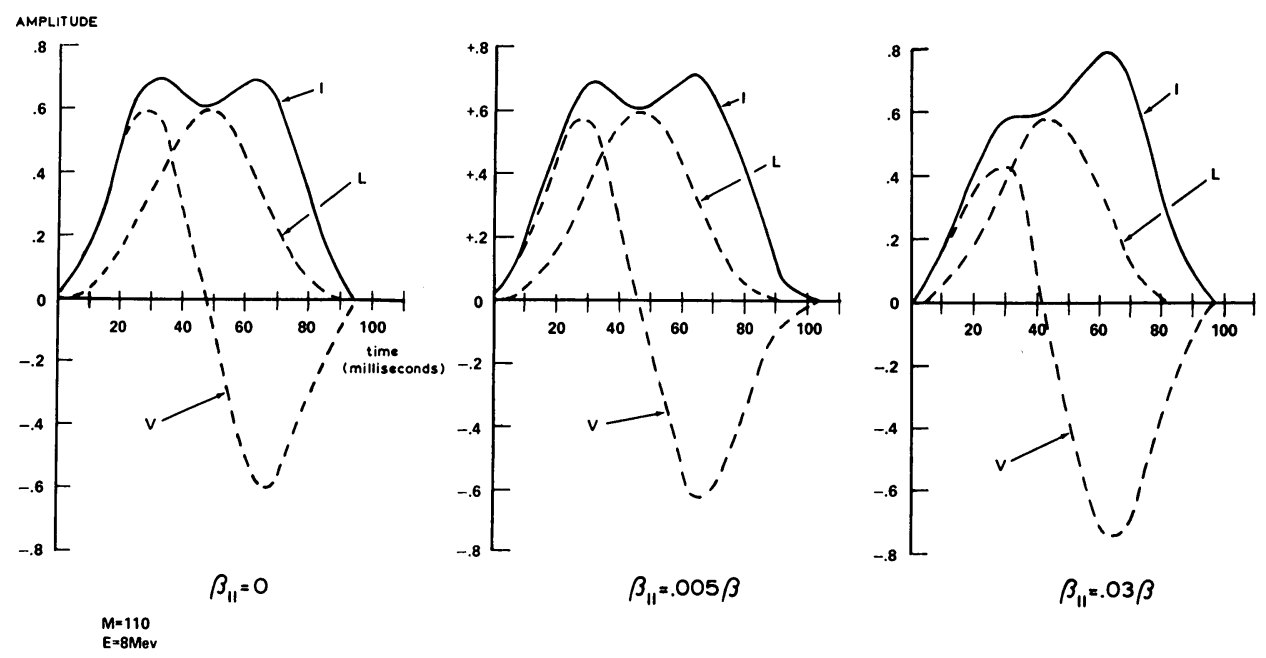

Fig. 2.

If charged particles are trapped at some radius, $R$, in the magnetosphere of a neutron star they may be heated by one or more mechanisms and thus exhibit a change of energy with time. Equation (1) is used to compute the pulse shapes that would be observed at a fixed receiver frequency if the energy of the charges $(\gamma)$ were changing with time in a region of constant magnetic field, $B$. If $\gamma$ increases with time, the fundamental synchrotron mode will vary as $e B / \gamma m_{0}$. Thus, if the radio receiver is turned to a fixed frequency, then if $\gamma$ changes to a value $\gamma_{2}$ from $\gamma_{1}$ a synchrotron mode $m_{2}$ is detected that is related to the initial value $m_{1}$ by the expression $m_{2}=m_{1} \gamma_{2} / \gamma_{1}$.

Thus, as $\gamma$ is changed in the computation, the pulse shape is computed for the new mode that corresponds to a constant receiver frequency. (Assumed equal to some initial value, $m$.) The results of such a computation for the case where $\beta_{\|}=0$ predicts a rather distinctive behavior for the pulse shapes. The peak to peak pulse separation for a double peaked pulsar is predicted to increase from zero to a saturation 
value with increasing energy. This result is illustrated in Figure 3 for a value of $\tau=1.871 \mathrm{sec}$ (CP 1133). The solid line represents the predicted peak to peak separation for a fixed receiver frequency as a function of energy. Note that as the energy of the emitting charges increases; (a) the overall pulse width, $\Delta \tau_{2}$ at the half power point decreases; (b) the ratio of the peak to the minimum of the double pulse increases and (c) the amplitude $A_{1}$ increases. Each synchrotron mode, (i.e. receiver-frequency) will generate a different curve - as illustrated by the dashed lines. The lower limit of the energy was arbitrarily set at $2 \mathrm{MeV}$ to conserve computation time.

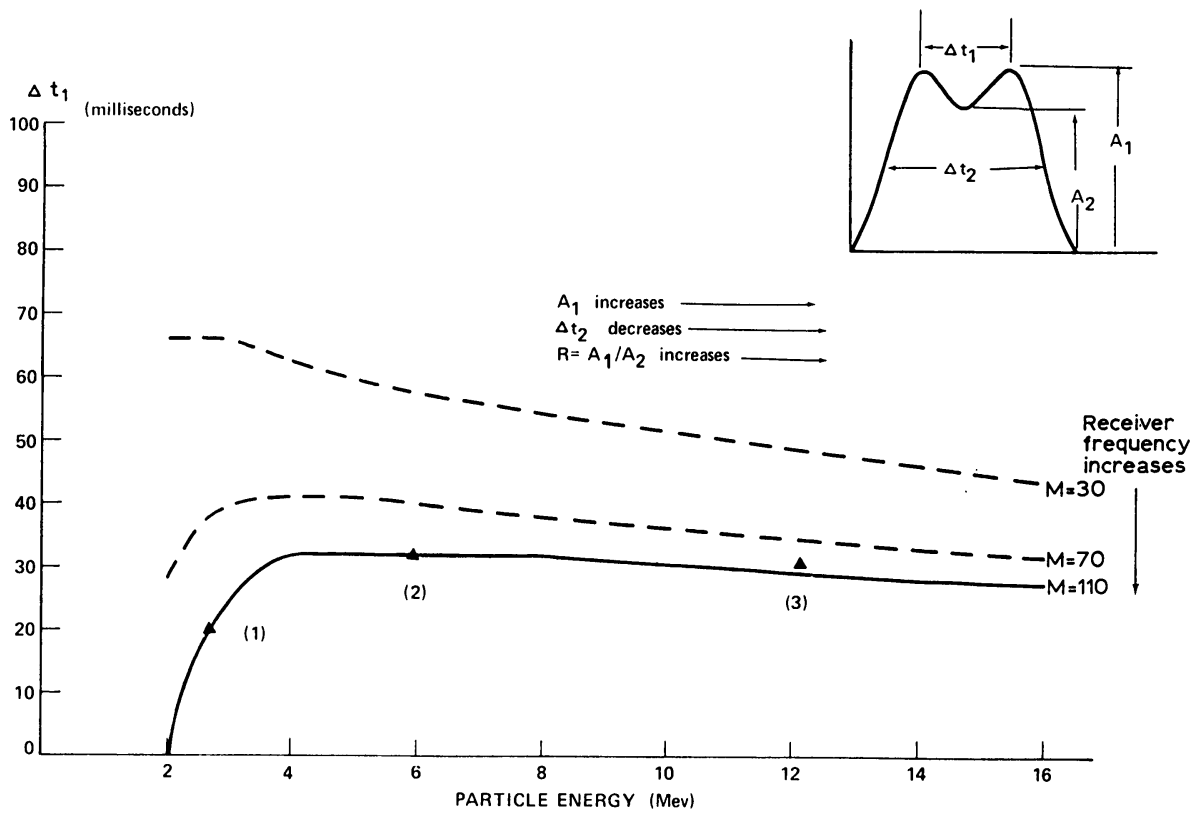

Fig. 3.

Such behavior seems to have been observed in sequential pulse data from CP 1133 (Craft, 1970). The peak to peak separation of three sequential pulses (1), (2) and (3) are plotted as triangles on Figure 3. (Note that due to the saturation of $\Delta t_{1}$ with energy, that the location of pulse (2) and (3) relative to the energy co-ordinate is somewhat arbitrary.) The data is compared with theoretical pulse shapes in Figure 4.

The upper row of curves are theoretically predicted shapes for three different particle energies. The lower curves are the three sequential pulses from Craft (1970) taken at a receiver frequency of $196.5 \mathrm{MHz}$. (A better comparison will be possible when Equation (1) can be completed for values of $m>950$.) Based on this data it appears that the rate of change of energy is close to $4.0 \mathrm{MeV} / \mathrm{sec}$. (The pulses occurred $1.187 \mathrm{sec}$ apart.) There is thus a possibility that this data represents a direct observation of increasing particle energy. Much additional comparison with data remains before the details of such energy gain are understood. For example, the 4 th pulse in the 

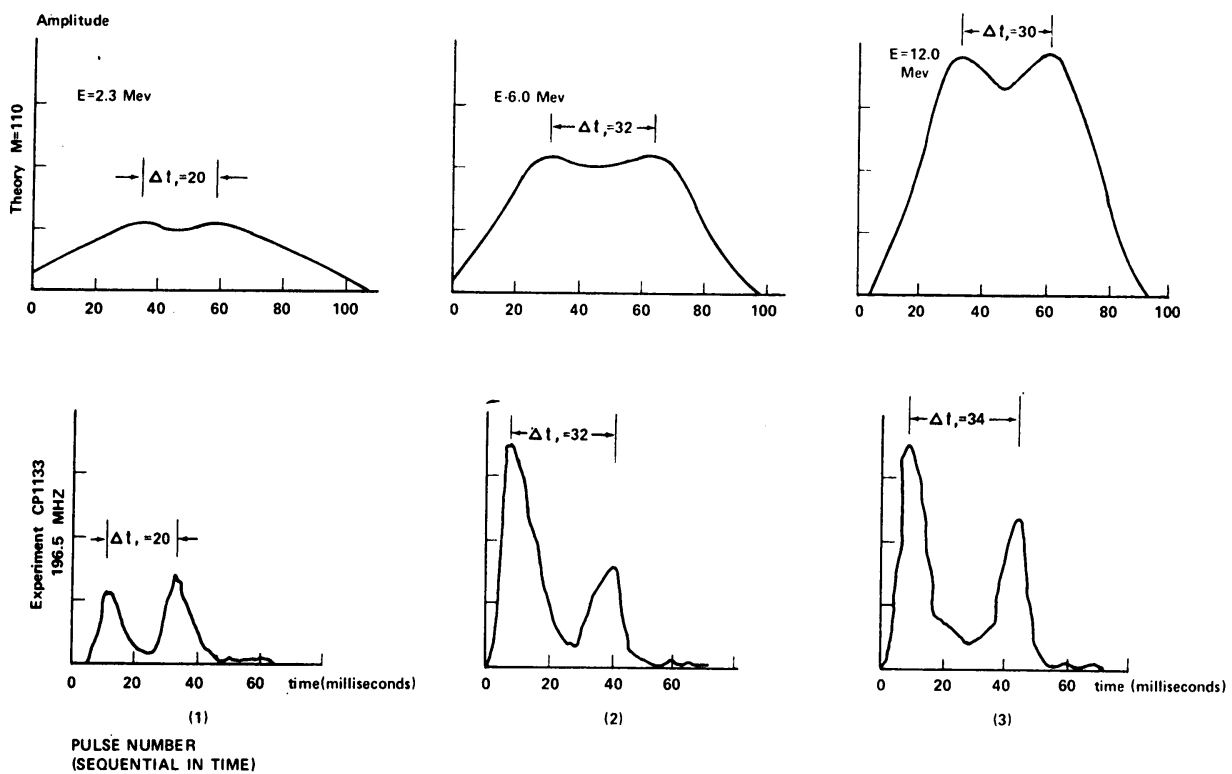

Fig. 4.

sequence was not observed. This could indicate that the energy emitted in coherent radiation by the bunched charges either exceeded the rate of energy gain or destroyed the coherence responsible for the emission. In general, the data from CP 1133 indicates a varying value for $\mathrm{d} \gamma / \mathrm{d} t$.

If such a heating effect is indeed occurring in some region of the magnetosphere, then this may have some consequences as far as the optical behavior of pulsars is concerned. The critical frequency of synchrotron emission (the frequency near the maxima of the spectrum) is given by the expression (Bekefi, 1966):

$$
\omega_{c}=\frac{3 e B}{2} \frac{e B}{m_{0}} \gamma^{2} .
$$

Thus, there will be appreciable optical emission only for large values of $\gamma$. This suggests a technique for finding optical signals in pulsars other than NP 0532. The individual pulse shapes of sequential pulses must be recorded and the energy of the charges emitting the radiation determined from the pulse shape. (The shape gives a more accurate value for $\gamma$, than the amplitude because the amplitude is a function of $\gamma$, $N^{2}$ and other factors affecting transmission of the signal.) If a set of simultaneous measurements are recorded, then only those recordings taken during pulses with large $\gamma$ should be added to obtain the optical signal. Such a measurement would require synchronization of an optical and radio telescope but could enhance the signal to noise ratio for optical searches by recording signals only during periods of optical activity. Note that the large values of $\gamma$ do not seem to occur with any predictable or regular pulse spacing. 
A crude power balance calculation can be performed on the basis of a theory by Grawe (1969) for RF heating in a magnetic mirror. The observed value of $\mathrm{d} E / \mathrm{d} t$ of $4 \times 10^{6} \mathrm{eV} / \mathrm{sec}$ can be accounted for by a heating flux of 700 to $7000 \mathrm{~W} / \mathrm{m}^{2}$ of RF power incident on the region of the magnetosphere in which the emitting charges are trapped. (The theories of Chiu and Occhionero (1969) or Kaplan and Tystovich (1970) could provide mechanisms tor such a flux of RF power.) If the emitting region is at a radius of $2 \times 10^{8} \mathrm{~cm}$, then a steady state power of $10^{16}-10^{17} \mathrm{~W}$ is indicated. This is within an order of magnitude of the observed $10^{18} \mathrm{~W}$ based on a beaming assumption and the data of Ekers and Moffet (1969).

In conclusion, the single particle formula, through detailed comparison with pulsar data seems to indicate effects due to mirroring and heating of charged particles. A method for detecting optical signals in other pulsars is suggested. Exact calculations of single particle pulse shapes as a function of $\gamma, \beta_{\perp}$ and $\beta_{\|}$are under way at the present time for synchrotron modes from the RF to the optical region and should be available soon.

The present calculations, based on the exact solution of the polar emission pattern for single particle synchrotron radiation represents merely the first step on the road to a complete theory. It is hoped that such computations will be found useful by others working on more complete analysis of pulsar phenomena.

\section{References}

Bekefi, G.: 1966, Radiation Processes in Plasma, John Wiley and Sons, New York.

Chiu, H. Y. and Occhionero, F.: 1969, Nature 223, 1113.

Clark, R. R. and Smith, F. G.: 1969, Nature 221, 724.

Craft, H. D., Jr.: 1970, 'Radio Observation of the Pulse Profiles and Dispersion Measures of 12 Pulsars'. Ph.D. Thesis, Cornell University (Fig. No. 5.28).

Drake, F. D. and Craft, H. D.: 1968, Nature 220, 231.

Eastlund, B. J.: 1968, Nature 220, 1293.

Eastlund, B. J.: 1970a, Nature 225, 430.

Eastlund, B. J.: 1970b, to be published in the proceedings of the Conference on Pulsars and High Energy Activity in Supernovae Remnants, Rome, 18-20 December 1969.

Ekers, R. D. and Moffet, A. T.: 1969, Nature 220, 756.

Grawe, H.: 1969, Plasma Physics 11, 151.

Kaplan, S. A. and Tystovich, V. N.: 1970, Lebedev Physical Institute, Preprint No. 16, Moscow. Maran, S. P. and Cameron, A. G. W.: 1968, Physics Today, August.

Nodvick, J. S. and Saxon, D. S.: 1954, Phys. Rev. 96, 180. 